\title{
Gerenciamento de resíduos sólidos nas unidades básicas de saúde de Picos-PI
}

Recebido em: 20/03/2013

Aprovado em: 16/05/2014

\author{
Eduardo Fernandes Oliveira' \\ Laura Maria Feitosa Formiga² \\ Luisa Helena de Oliveira Lima ${ }^{3}$ \\ Bartira Bezerra de Brito ${ }^{4}$ \\ Raíla Carvalho Feitosa ${ }^{5}$
}

Resumo: Objetivou-se avaliar as medidas realizadas nas unidades de saúde de Picos - PI, para o gerenciamento dos resíduos sólidos. Foi desenvolvido um estudo transversal de abordagem quantitativa com 19 enfermeiros mediante aplicação de um formulário semi-estruturado. Os resultados evidenciam que $63,2 \%$ possuíam o plano de gerenciamento de resíduos, $89,5 \%$ segregavam os resíduos perfurocortantes, 52,6\% os resíduos químicos, $73,7 \%$ os resíduos potencialmente contaminados e $68,4 \%$ os resíduos comuns. Destaca-se a ausência de um plano formal para o gerenciamento dos resíduos produzidos em algumas unidades, sendo este documento indispensável para o manejo correto dos resíduos.

Descritores: Gerenciamento de Resíduos; Resíduos Sólidos; Centros de saúde

\section{Solid waste management in health basic units of Picos -PI}

Abstract: This study aimed to evaluate the measures undertaken in the health units of Picos - PI for the management of solid waste. It was developed a cross-sectional study with a quantitative approach with 19 nurses by applying a semi-structured form. The results showed that $63.2 \%$ had a waste management plan, $89.5 \%$ segregated waste sharps, $52.6 \%$ chemical residues, $73.7 \%$ potentially contaminated waste and $68.4 \%$ common waste. We highlight the lack of a formal plan for managing the waste produced in some units, this being essential document to the correct management of waste. Descriptors: Waste Management; Solid Waste; Health Centers.

\section{Gestión de residuos sólidos en las unidades básicas de salud de Picos -PI}

Resumen: Este estudio tuvo como objetivo evaluar las medidas adoptadas en las unidades de salud de Picos - PI para el manejo de residuos sólidos. Se desarrolló un estudio transversal con enfoque cuantitativo con 19 enfermeras mediante la aplicación de un formulario semi-estructurado. Los resultados mostraron que el 63,2\% tenía un plan de gestión de residuos, 89,5\% segregada residuos cortopunzantes, 52,6\% de residuos químicos, 73,7\% residuos potencialmente contaminados y $68,4 \%$ de residuos común. Cabe destacar la ausencia de un plan formal para la gestión de los residuos producidos en algunas unidades, este documento es esencial para la correcta gestión de los residuos.

Descriptores: Administración de Residuos; Residuos Sólidos; Centros de Salud.

\section{INTRODUÇÃO}

A problemática da geração de lixo já preocupa as autoridades sanitárias há algum tempo. Particularmente, o lixo produzido por serviços de saúde acentua os riscos à saúde da população bem como ao meio ambiente, tendo em vista seu alto risco de contaminação. Atualmente os lixos produzidos por prestadores de serviço de saúde são denominados de resíduos de serviços de saúde (RSS).

Os grandes geradores possuem maior consciência a respeito do planejamento adequado e necessário para o gerenciamento dos RSS. Contudo, os pequenos geradores muitas vezes não possuem essa consciência nem os conhecimentos necessários ${ }^{(1)}$.

O gerenciamento dos RSS constitui-se em um conjunto de procedimentos de gestão, planejados e implementados a partir de bases científicas e técnicas, normativas e legais, com o objetivo de minimizar a produção de resíduos e proporcionar, aos resíduos gerados, um encaminhamento seguro, de forma eficiente, visando a proteção dos trabalhadores, a preservação da saúde, dos recursos naturais e do meio ambiente. Deve abranger todas as etapas de planejamento dos recursos físicos, dos recursos materiais e da capacitação dos recursos humanos envolvidos no manejo de RSS(2).

O Plano de Gerenciamento dos Resíduos de Serviços de Saúde (PGRSS) é o documento que aponta e descreve as ações relativas ao manejo de resíduos sólidos, que corresponde às etapas de: segregação, acondicionamento, coleta, armazenamento, transporte, tratamento e disposição final. Deve considerar as características e riscos dos resíduos, as ações de proteção à saúde e ao meio ambiente e os princípios da biossegurança de empregar medidas técnicas, administrativas e normativas para prevenir acidentes ${ }^{(2)}$.

O gerenciamento dos resíduos sólidos no âmbito hospitalar sempre teve mais destaque com maior número de publicações, enquanto em unidades básicas de saúde o assunto é pouco discutido, sendo esse tipo de serviço um produtor considerável de resíduos sólidos. As falhas ocorridas no gerenciamento desses resíduos podem acarretar problemas ambientais e na própria saúde do trabalhador.

Em virtude disso, o presente estudo teve como objetivo avaliar as medidas realizadas para o gerenciamento dos resíduos sólidos, nas unidades de saúde de Picos - PI.

\section{METODOLOGIA}

Realizou-se um estudo transversal de cunho exploratóriodescritivo, com abordagem quantitativa nas unidades de saúde da família do município de Picos-PI.

Como critério de inclusão da unidade na pesquisa, a equipe de saúde da família deve ter o profissional enfermeiro cadastrado 
durante a fase de coleta dos dados e está localizado na zona urbana.

No período da coleta de dados, uma unidade básica de saúde não possuía o enfermeiro como gerente, não atendendo o critério de inclusão, com isso a população e amostra foram compostas por 19 enfermeiros.

A coleta de dados ocorreu, entre agosto e setembro de 2011, através da aplicação de um formulário contendo dados como: existência de plano de gerenciamento de resíduos sólidos, manipulação específica dos resíduos e se há segregação de resíduos sólidos.

A pesquisa foi aprovada pelo Comitê de Ética da Universidade Federal do Piaui( ${ }^{\prime 3}$. A análise dos dados foi possível após a construção de tabelas, a partir dos dados tabulados, com o auxílio do software Excel versão 2007 e o programa SPSS versão 17.0.

\section{RESULTADOS E DISCUSSÃO}

Os resultados desse estudo mostram que os enfermeiros gerentes das UBS eram predominantemente do sexo feminino, com idade entre 25 e 29 anos de idade e tempo de serviço entre 3 a 5 anos e a maioria possuía especialização, conforme a tabela 1.

Fato historicamente comprovado por vários estudos, como o que avaliou a implantação do plano de gerenciamento dos resíduos dos serviços de saúde nas unidades de Saúde do Distrito Sanitário II do Município de Olinda, com 90\% de participação feminina. Se diferenciando apenas com relação a média de idade e tempo de serviço, no qual a média de idade dos enfermeiros era de 34 anos e tempo de serviço nas instituições 9,4 anos. Em geral, os trabalhadores são jovens e possuem tempo suficiente para conhecer as rotinas das instituições ${ }^{(4)}$.

Tabela 1 - Perfil dos enfermeiros participante da pesquisa. PicosPI, set/out. 2011

\begin{tabular}{|c|c|c|}
\hline $\begin{array}{l}\text { Variáveis relacionadas ao } \\
\text { perfil dos enfermeiros }\end{array}$ & $\mathbf{n}$ & $\%$ \\
\hline \multicolumn{3}{|l|}{ Sexo } \\
\hline Masculino & 2 & 10,5 \\
\hline Feminino & 17 & 89,5 \\
\hline \multicolumn{3}{|l|}{ Idade (em anos) } \\
\hline De 25 à 29 & 8 & 42,1 \\
\hline De 30 a 35 & 6 & 31,6 \\
\hline De 36 a 40 & 2 & 10,5 \\
\hline De 46 a 50 & 3 & 15,8 \\
\hline \multicolumn{3}{|l|}{$\begin{array}{l}\text { Tempo de serviço em ESF } \\
\text { (em anos) }\end{array}$} \\
\hline De0a 1 ano & 1 & 5,3 \\
\hline De 1 a 3 anos & 6 & 31,6 \\
\hline De 3 a 5 anos & 4 & 21,1 \\
\hline De 5 a 10 anos & 6 & 31,6 \\
\hline De 10 a 15 anos & 2 & 10,5 \\
\hline \multicolumn{3}{|l|}{ Especialização } \\
\hline Sim & 16 & 84,2 \\
\hline Não & 3 & 15,8 \\
\hline
\end{tabular}

Sobre as atividades desenvolvidas nas Unidades Básicas de saúde, observou que em apenas $6(31,6 \%)$ UBS possuíam atendimentos de imunização. Observou-se ainda a presença de centrais de material esterilizado em 18 unidades. Fato semelhante relatado no estudo ${ }^{(4)}$ onde todas as unidades possuíam central de esterilização diferenciando na questão no número de sala de vacina onde todas as UBS ofereciam imunização.

Foram identificadas, dentre as atividades desenvolvidas nas unidades, aquelas com o potencial de gerar resíduos, obtendo-se o seguinte resultado: em 17 atendimentos médico, em 18 odontológicos e em todas as 19 unidades pesquisadas eram realizados curativos, exames de prevenção do câncer do colo do útero e possuíam farmácia básica, com a dispensação dos medicamentos prescritos.

Tais atividades, exercidas nas UBS pesquisadas, geram diferentes tipos de resíduos, classificados de acordo com a Resolução n 33 de 2003 da ANVISA em:

Grupo A - resíduos potencialmente infectantes. Na UBS são considerados resíduos deste grupo vacinas de microrganismos vivos ou atenuados.

Grupo B - resíduos químicos. Podem ser considerados resíduos químicos nas UBS medicamentos antibacterianos vencidos de uso sistêmico ou tópico, desinfetantes, além dos resíduos das substâncias químicas utilizadas no serviço de odontologia.

Grupo D - resíduos comuns. Nas UBS são considerados resíduos comuns: luvas, esparadrapo, algodão, gazes, equipos e materiais similares, que tenham entrado em contato ou não com sangue, tecidos ou fluidos orgânicos; papéis de uso sanitário; papéis de uso administrativo; resíduos da varrição; materiais passíveis de reciclagem; embalagens em geral.

Grupo E - resíduos perfuro-cortantes. Nas UBS estão enquadrados neste grupo agulhas e escalpes, lâminas de bisturi e ampolas de vidro.

De acordo com tabela 2, No que se refere ao plano de gerenciamento de resíduos, observou-se que 12 UBS possuíam o plano de gerenciamento de resíduos. Diferentemente de outra pesquisa(5), realizada em 49 UBS onde em nenhuma instituição foi encontrada o plano de gerenciamento.

O responsável pelo estabelecimento gerador deverá implementar um PGRSS, definido como um conjunto de procedimentos de gestão, planejados e implementados baseando-se em normas científicas, normativas e legais, com o objetivo de minimizar a produção e proporcionar aos resíduos gerados um encaminhamento seguro, de forma eficiente, visando a proteção dos funcionários, a preservação da saúde pública, dos recursos naturais e do meio ambiente ${ }^{(1)}$.

Uma cópia do PGRSS deve estar disponível para consulta sob solicitação da autoridade sanitária ou ambiental competente, dos funcionários, dos pacientes e do público em geral ${ }^{(2)}$.

Tabela 2 - Gerenciamento dos resíduos. Pico-PI, set/out. 2011

\begin{tabular}{lcc}
$\begin{array}{l}\text { Variáveis relacionadas } \\
\text { ao gerenciamento dos } \\
\text { resíduos (n=19) }\end{array}$ & $\mathbf{n}$ & $\%$ \\
\hline Plano de gerenciamento & 12 & 63,2 \\
Sim & 7 & 36,8 \\
\hline Não & 17 & \\
\hline Segregação dos resíduos & 2 & 89,5 \\
\hline Sim & 17 & 10,5 \\
Não & 10 & 89,5 \\
Quais de resíduos & & 52,6 \\
\hline Perfurocortantes &
\end{tabular}




\begin{tabular}{|c|c|c|}
\hline $\begin{array}{l}\text { Resíduos potencialmente } \\
\text { contaminados }\end{array}$ & 14 & 73,7 \\
\hline Resíduos comuns & 13 & 68,4 \\
\hline \multicolumn{3}{|l|}{ Acondicionamento } \\
\hline Resíduos tipo A & 14 & 73,7 \\
\hline Resíduos tipo B & 10 & 52,6 \\
\hline Resíduos tipo D & 13 & 73,7 \\
\hline Resíduos tipo $\mathrm{E}$ & 17 & 89,5 \\
\hline \multicolumn{3}{|c|}{$\begin{array}{l}\text { Transporte interno carro } \\
\text { adaptado }\end{array}$} \\
\hline Sim & 13 & 68,4 \\
\hline Não & 6 & 31,6 \\
\hline \multicolumn{3}{|c|}{ Quanto o armazenamento } \\
\hline Há local especifico & 12 & 63,2 \\
\hline Não possui & 7 & 36,8 \\
\hline \multicolumn{3}{|c|}{$\begin{array}{l}\text { Órgão público recolhe os } \\
\text { resíduos }\end{array}$} \\
\hline Sim & 18 & 94,7 \\
\hline Não & 1 & 5,3 \\
\hline \multicolumn{3}{|l|}{ EPI } \\
\hline Luvas & 18 & 94,7 \\
\hline Botas & 16 & 84,2 \\
\hline Mácaras & 12 & 63,2 \\
\hline Avental & 13 & 68,4 \\
\hline
\end{tabular}

Na questão de segregação dos resíduos, verificou-se que $89,5 \%$ das UBS segregavam os resíduos, sendo os resíduos perfuro-cortantes os mais segregados (89,5\%).

Outra pesquisa(5), apresentou um percentual elevado na segregação dos perfuro cortante totalizando $95 \%$ do total das UBS pesquisadas. Diferindo na segregação dos resíduos químicos onde alcança $85 \%$ da amostra pesquisada, e se assemelha na segregação dos resíduos comum e potencialmente infectante.

A segregação é o ponto fundamental de toda a discussão sobre a periculosidade ou não dos resíduos de serviços de saúde. Apenas uma parcela é potencialmente infectante, contudo, se ela não for segregada, todos os resíduos que a ela estiverem misturados também deverão ser tratados como potencialmente infectantes, exigindo procedimentos especiais para acondicionamento, coleta, transporte e disposição final, elevando assim os custos do tratamento desses resíduos ${ }^{(1)}$.

A segregação é uma das operações fundamentais para permitir o cumprimento dos objetivos de um sistema eficiente de manuseio de resíduos e consiste em separar ou selecionar apropriadamente os resíduos segundo a classificação adotada. Essa operação deve ser realizada na fonte de geração e está condicionada à prévia capacitação do pessoal de serviço ${ }^{(2)}$.

Quanto ao acondicionamento dos resíduos, observou-se que $68,7 \%$ acondicionavam os resíduos do tipo $A$ e $D, 73,7 \%$ os resíduos do tipo $B$, e $89,5 \%$ do tipo $E$.

$\mathrm{O}$ acondicionamento deve ser executado no momento e no local de sua geração, em recipientes adequados a cada tipo, quantidade e característica, de acordo com as normas estabelecidas pela NBR 12.808(6), evitando sua exposição e consequentemente diminuindo os riscos de contaminação.

O acondicionamento de resíduo sólido de serviços de saúde (RSS) em sacos plásticos diferenciados auxilia o gerenciamento correto do resíduo, levando todos os profissionais que trabalham no estabelecimento a prestarem atenção a esta questão, chegando a detectar problemas, quando existirem ${ }^{(7)}$.

O RSS classificado como comum, pela NBR 12.808, pode ser acondicionado em sacos tipo 1, podendo ter qualquer cor. $\mathrm{O}$ resíduo infectante deve, pelas normas técnicas aplicáveis, ser acondicionado em sacos plásticos tipo 2, de cor branca-leitosa. Deve constar, individualmente, a identificação do fabricante e o símbolo de material infectante ${ }^{(6-8)}$.

O RSS perfuro-cortante deve ser acondicionado, em recipientes apropriados para evitar acidentes. Pode ser qualquer recipiente que atenda às características de resistência à perfuração, estanqueidade e impermeabilidade. É recomendável que as agulhas não sejam removidas das seringas, após o uso, e que sejam descartadas diretamente em recipiente próprio ${ }^{(8)}$.

Das 19 UBS, 68,4\% possuíam carro adaptado para o transporte dos resíduos, $63,2 \%$ possuíam locais específicos para armazenamento dos resíduos e em $94,7 \%$ a coleta era realizada por órgão público. Algo semelhante encontrado noutra pesquisa(9), onde 78,9\% possuíam carro adaptado especifico para esse fim e em todas as unidades possuía local para o armazenamento e todas as unidades algum órgão público recolhia os resíduos regulamente.

O transporte deve ser realizado em veículos ou carrinhos específicos para este serviço, dimensionados de acordo com o volume coletado. Devem ser de material liso, sem arestas e com tampa e ser lavados e higienizados após a coleta(10).

Quanto ao uso de EPI para manipulação dos resíduos notou-se que em $94,7 \%$ das unidades faziam uso de luvas, $84,2 \%$ de botas, $63,2 \%$ de máscaras e $68,4 \%$ de avental, o que corrobora achados de outros estudos(9). Pode se analisar que o uso de EPI no manuseio dos resíduos é parcial podendo acarretar problemas para a saúde do trabalhador.

A NBR 12.809/9314 estabelece que para o manuseio dos resíduos infectantes, as pessoas devam usar equipamentos de proteção individual, como: gorro, óculos, máscara, avental, luvas e botas. Vale ressaltar, que no manuseio e transporte dos RSS é exigido o uso do conjunto de EPI's, o que não se configura como verdade na prática das unidades analisadas.

Os equipamentos de proteção devem ser utilizados pelos funcionários que manuseiam os resíduos e devem ser os mais adequados para lidar com os tipos de resíduos de serviços de saúde. Devem ser utilizados de acordo com as recomendações normativas do Ministério do Trabalho.

Quanto ao treinamento da equipe para manipulação dos resíduos, verificou-se que 8 das equipes tinham algum membro capacitado. A maior participação no treinamento foi do enfermeiro $(31,6 \%)$, seguido pelo auxiliar de serviços gerais (26,3\%).

O programa de educação continuada, previsto na RDC ANVISA no 306/04, visa orientar, motivar, conscientizar e informar permanentemente a todos os envolvidos sobre os riscos e procedimentos adequados de manejo, de acordo com os preceitos do gerenciamento de resíduos. De acordo com a RDC ANVISA no 306/04, os serviços geradores de RSS devem manter um programa de educação continuada, independente do vínculo empregatício dos profissionais ${ }^{(2)}$.

Treinamento dos funcionários para a correta segregação dos resíduos é bastante compensador, pois resulta no encaminhamento para coleta, tratamento e disposição final especial apenas dos resíduos que realmente necessitam desses procedimentos, reduzindo as despesas com o tratamento ao mínimo necessário(1).

Em relação às facilidades, as mais relatadas foram: disponibilidade de recipiente adequado para resíduos perfuro-cortantes e 
existência na UBS de local adequado para armazenar os resíduos (73,7\%), seguido de característica dos resíduos gerados (pouco volume e capacidade infectante) e disponibilidade de transporte para o lixo contaminado, com 57,9\% cada.

No que diz respeito às dificuldades encontradas para o gerenciamento resíduos, 57,9\% dos enfermeiros marcaram a ausência de treinamento como maior empecilho para atividade de gerenciar os resíduos, seguido de ausência de autoclave para inativação de imunobiológicos (36,8\%).

\section{CONCLUSÃO}

Pode-se observar que o gerenciamento dos resíduos sólidos possui diversas falhas, desde a segregação, acondicionamento até o armazenamento, e isso deve se pela incipiência do treinamento da equipe para o gerenciamento e também por falta de um plano formal para o gerenciamento de resíduos.

As informações do estudo podem melhorar o planejamento das ações do gerenciamento e também servir como fonte de pesquisa para os gerentes das unidades básicas de saúde para desenvolver o plano de gerenciamento, documento necessário e obrigatório para manipulação correta do gerenciamento dos resíduos.

Dentre o acondicionamento de resíduos, o mais adequado manejo encontra-se no destino dos perfuro-cortantes, tendo em vista que é uma importante fonte de infecção. O presente estudo tem o propósito de incentivar discussões acerca da importância de uma atuação mais segura, sensibilizando quanto aos riscos e identificação de situações que contribuam para a prevenção de acidentes.

\section{Referências}

1. Garcia LPP, Ramos BGZ. Gerenciamento dos resíduos de serviço de saúde: Uma questão de biossegurança. Cad. Saúde Pública. 2004; 20(3): 744-752.

2. Ministério da Saúde (BR); Agência Nacional de Vigilância

Sanitária - Manual de gerenciamento de resíduos de serviços de saúde. Brasília (Brasil): Ministério da Saúde; 2006.

3. Ministério da Saúde (BR); Conselho Nacional de Saúde.

Resolução №196/96 - Normas regulamentadoras de pesquisa envolvendo seres humanos. Brasília (Brasil): Ministério da Saúde; 1996.

4. Silva CA, Madeira IEAF, Lima RA. Avaliação da Implantação do Plano de Gerenciamento dos Resíduos dos Serviços de Saúde nas Unidades de Saúde do Distrito Sanitário II do Município de Olinda [monograph]. Recife: Departamento de Saúde Coletiva, Centro de Pesquisas Aggeu Magalhães, Fundação Oswaldo Cruz; 2009. 42 p.

5. Almeida VCF, Pinto SL, Nascimento AJR, Feitosa CR, Alencar

PRP. Gerenciamento dos resíduos sólidos em unidade de saúde da família. Rev. Rene. Fortaleza. 2009; 10(2): 103-112.
6. Associação Brasileira de Normas e Técnicas. NBR 12808: resíduo de serviço de saúde: classificação. Rio de Janeiro, 1993.

7. Maragoni MC. Gerenciamento de resíduo de serviço de saúde: estudo de caso hemocentro da Unicamp [dissertacion] Campinas: Comissão de Pós-graduação da Faculdade de Engenharia Civil, Arquitetura e Urbanismo da Universidade Estadual de Campinas, Unicamp; 2006. 114p.

8. Schneider VE, Emmerich RC, Duarte VC, Orlandin SM. Manual de gerenciamento de resíduos sólidos em serviços de saúde. 2 ed. Caxias do Sul: Educs; 2004.

9. Serra A, Castro JP, Sousa MCB, Ribeiro MLH, Santos AM. Gerenciamento dos resíduos sólidos dos serviços de saúde em unidades mistas do município de São Luís-Ma. Revista do hospital universitário

10. Associação Brasileira de Normas e Técnicas. NBR 12.810: coleta de resíduo de serviço de saúde: procedimento. Rio de Janeiro, 1993. 